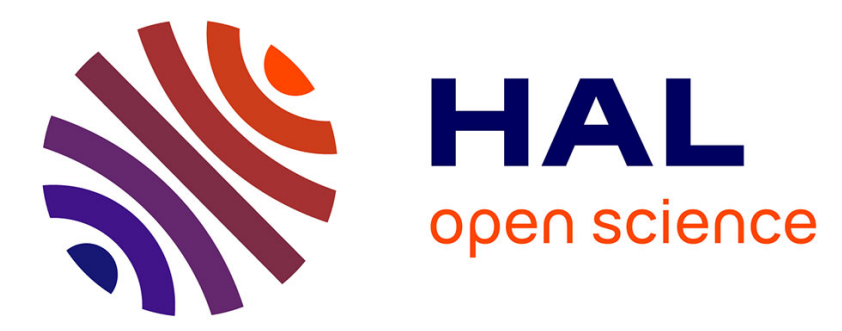

\title{
Nonlinear Dual Mode Adaptive Control of PAR2 : a 2-dof Planar Parallel Manipulator, with Real-time Experiments
}

Guilherme Sartori Natal, Ahmed Chemori, François Pierrot, Olivier Company

\section{- To cite this version:}

Guilherme Sartori Natal, Ahmed Chemori, François Pierrot, Olivier Company. Nonlinear Dual Mode Adaptive Control of PAR2 : a 2-dof Planar Parallel Manipulator, with Real-time Experiments. IROS: Intelligent Robots and Systems, Oct 2009, St. Louis, MO, United States. pp.2114-2119, 10.1109/IROS.2009.5354083 . lirmm-00429818

\section{HAL Id: lirmm-00429818 https://hal-lirmm.ccsd.cnrs.fr/lirmm-00429818}

Submitted on 4 Nov 2009

HAL is a multi-disciplinary open access archive for the deposit and dissemination of scientific research documents, whether they are published or not. The documents may come from teaching and research institutions in France or abroad, or from public or private research centers.
L'archive ouverte pluridisciplinaire HAL, est destinée au dépôt et à la diffusion de documents scientifiques de niveau recherche, publiés ou non, émanant des établissements d'enseignement et de recherche français ou étrangers, des laboratoires publics ou privés. 


\title{
Nonlinear Dual Mode Adaptive Control of PAR2 : a 2-dof Planar Parallel manipulator, with Real-time experiments
}

\author{
G. Sartori Natal and A. Chemori and F. Pierrot and O. Company
}

\begin{abstract}
This paper deals with nonlinear dual mode adaptive control of a redundant manipulator for a pick-andplace scenario with high acceleration (20G). For performance comparisons, a conventional Proportional-Derivative (PD) controller has also been implemented. In this context, the experimental testbed is not equipped with velocity sensors. Therefore, a high-gain observer has been implemented to estimate the articular velocities. Real-time experiments show the performance improvements obtained by the proposed control approach in comparison to the conventional one.
\end{abstract}

\section{INTRODUCTION}

Par2 is a new two-degree-of-freedom ( $d o f$ ) parallel manipulator that is able to produce two translations in the vertical plane, and can reach accelerations above 40G. The proposed architecture of this robot can guarantee that it can be lighter and stiffer than a classical 2 dof planar mechanism [13]. One other advantage of this parallel robot is that it has the possibility to keep the motors fixed on the base, allowing fast movements to be performed [7].

In order to achieve such accelerations and perform an accurate movement, a good controller must be used. By using simple linear single-axis controllers (such as a Proportional Derivative (PD) controller), the tracking performance can be limited, especially when the robot has highly nonlinear dynamics and/or when the velocities/accelerations are high [7].

During last decades, adaptive control systems have been widely developed. The main principle of this control technique is to adjust automatically the controller parameters in the case of unknown and time-varying process parameters in the aim that a desired degree of the performance index is met. One of the main characteristics of adaptive control systems is their capability to tune the controller parameters in real-time from the measurable information of the closedloop system.

In the literature, there are mainly four techniques of adaptive control, namely Gain Scheduling, Model Reference Adaptive Control, Self-tuning controllers and Dual control. They can be classified into two classes: Direct methods and indirect methods. In the first case, the adjustment rules tell us directly how the controller parameters should be updated. This class of schemes includes: Gain scheduling [15] and Model Reference Adaptive Control [10].

This work is supported by Objectif 100G: a French National Project on Parallel Robots.

G. Sartori Natal, A. Chemori, F. Pierrot and O. Company are with LIRMM, Univ. Montpellier 2 - CNRS, 161 rue Ada, 34392 Montpellier, France sartorinatalelirmm. fr
In the former, a set of measurable variables that correlate with eventual changes in the process dynamics is used to adapt the controller parameters. The latter was proposed to solve the problem in which the performance specifications are given in terms of a reference model.

The second class concerns indirect methods, where the estimates of the process parameters are updated and the controller parameters are obtained through the solution of a design problem using estimated parameters. This class includes: Self-tuning controllers [1] and Dual control [5].

The three first adaptive controllers do not take into account parameter uncertainties in the design stage. However, if nonlinear stochastic control theory is used, it will significantly improve the performance of the control approach. Already in 1960, A. Feldbaum indicated that adaptive control systems based on the certainty-equivalence (CE) approach are often far away from being optimal. Instead of the CE approach, he introduced the principle of adaptive dual control [5]. The design of such a controller takes into account uncertainties in the estimated parameters, so that it will be able to take special actions when it has poor knowledge about the system.

Different model-based adaptive controllers have been used until now for the control of parallel robots. In [7], a nonlinear adaptive feedforward controller was proposed for the control of the Hexaglide (a 6 dof parallel robot), in addiction to a PD feedback term. The main objective of this work was to show the convergence of the adaptive parameters by simulation. In [6], a scheme similar to the so called computed torque controller ${ }^{1}$ [4] was also proposed to control the Hexaglide robot, but with experimental results that showed a good improvement in the trajectory tracking with relation to a PD controller, although no control signal has been presented.

In this paper, a nonlinear Dual Mode (DM) controller, originally refered as 'binary' in [8], is proposed to control the Par2 parallel manipulator for a pick-and-place application with high acceleration. This controller consists in the one proposed by Slotine and $\mathrm{Li}$ in [16], with the addition of a projection to the parametric adaptation law, such that it would be possible to guarantee that the estimated parameters would be bounded.

This paper is organized as follows. In section 2, a description of the Par2 parallel robot is presented. Section 3 details the proposed control algorithm. In section 4, the experimental results are presented and commented. In section 5, a conclusion about the current results and the

\footnotetext{
${ }^{1}$ With the modification that the dynamic model is computed from the desired values, instead of the actual joint coordinates.
} 
expectations on the future performance of Par2 robot with the proposed controller is made.

\section{PAR2 PROTOTYPE: DESCRIPTION AND DYNAMICS}

\section{A. Description of the Par2 robot}

In the aim of application of the proposed control scheme, the prototype Par2 will be used. It's a two-dof parallel manipulator, illustrated in figure 1, with the following characteristics:

- the platform (6) is a rigid body,

- only the two inner arms (3) are actuated,

- the two other arms (4) are linked to the frame (1) through passive revolute joints,

- inner arms (3) and (4) are connected to the platform (6) with pairs of rods (5) mounted on ball joints (7),

- the rotations of the arms (4) are coupled in order to guarantee planar motions along $\mathrm{x}$ and $\mathrm{z}$ axes.
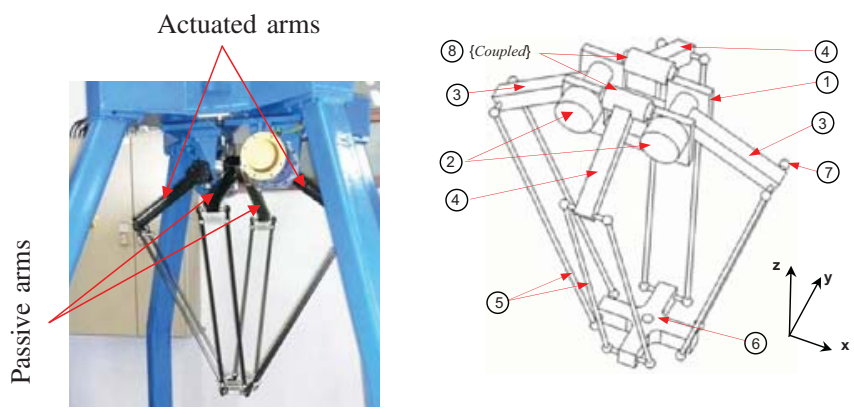

Fig. 1. The two-dof parallel manipulator Par2: view of the robot (left), schematic view of its mechanical structure (right)

The proposed architecture of the prototype has the following advantages:

- the two coupled passive chains made a constrained system supporting almost all the moments and force besides the driving forces,

- it can be designed with existing technologies and parts (can be made as light as for Delta [3] or Par4 [11] parallel robots)

- it is symmetric with respect to motion plane and the yoz plane in its centered position

The proper functioning of this two-dof parallel manipulator is guaranteed by the coupling of the rotation of arms (4). This constrains the robot's platform to evolve in one plane. The coupling means that the rotation of the first arm in the clockwise direction involves the rotation of second one in the counterclockwise direction.

Remark 1: The two actuated articulations of the robot are equipped with TPM50 Alpha motors coupled with gears having a reduction ratio of 21 . For more details about the prototype Par2, the reader is referred to [13].

\section{B. Dynamic model of the robot Par2}

The Lagrangian nonlinear dynamic model [14], [18] of the manipulator Par2 is given by:

$$
\begin{aligned}
& I_{e q}\left[\begin{array}{c}
\ddot{q}_{1} \\
\ddot{q}_{2}
\end{array}\right]=\tau-f_{d}\left[\begin{array}{c}
\operatorname{sign}\left(\dot{q}_{1}\right) \\
\operatorname{sign}\left(\dot{q}_{2}\right)
\end{array}\right]-f_{v}\left[\begin{array}{c}
\dot{q}_{1} \\
\dot{q}_{2}
\end{array}\right]- \\
& \frac{g}{2}\left(M_{1}+M_{2}\right)\left[\begin{array}{c}
L_{1} \cos \left(q_{1}\right) \\
L_{2} \cos \left(q_{2}\right)
\end{array}\right]+J^{T}\left(M_{p} \dot{J}(q, \dot{q}) \dot{q}-g\left[\begin{array}{c}
M_{p} \\
M_{p}
\end{array}\right]\right)
\end{aligned}
$$

where $I_{e q}=I_{d r v}+I_{f}+I_{a}+J^{T} M_{p} J, I_{d r v}$ is the motor driver inertia, $I_{f}$ is the forearm inertia, $I_{a}$ is the arm inertia, $J$ is the jacobian matrix, $M_{p}$ is the mass of the platform of the robot, $g$ is the gravitational acceleration, $f_{v}$ is the viscous friction coefficient, $f_{d}$ is the dry friction coefficient, $M_{1}$ and $M_{2}$ are the masses of the arms, $L_{1}$ and $L_{2}$ are their lengths.

This dynamic model can be written in the following matrix form:

$$
D(q) \ddot{q}+C(q, \dot{q}) \dot{q}+G(q)+f(q, \dot{q})=\tau
$$

where

$D(q) \in \mathbb{R}^{2 \times 2}$ is the inertia matrix,

$C(q, \dot{q}) \in \mathbb{R}^{2 \times 2}$ is the matrix of the centrifugal and Coriolis terms,

$G(q) \in \mathbb{R}^{2}$ is the vector of gravitational forces, $f(q, \dot{q}) \in \mathbb{R}^{2}$ is the vector of friction forces,

$\tau \in \mathbb{R}^{2}$ is the vector of control inputs (torques generated by the actuators),

$q=\left[\begin{array}{l}q_{1} \\ q_{2}\end{array}\right] \in \mathbb{R}^{2}$ is the vector of articular positions,

$\dot{q}=\left[\begin{array}{c}\dot{q}_{1} \\ \dot{q}_{2}\end{array}\right] \in \mathbb{R}^{2}$ is the vector of articular velocities,

$\ddot{q}=\left[\begin{array}{c}\ddot{q}_{1} \\ \ddot{q}_{2}\end{array}\right] \in \mathbb{R}^{2}$ is the vector of articular accelerations.

\section{PROPOSED SCHEME: A NONLINEAR ADAPTIVE DUAL MODE CONTROLLER}

The Dual Mode controller consists basically in the utilization of a high adaptation gain together with a projection of the estimated parameters. Then, to large tracking errors in the transitory stage, the controller behaves approximately as a sliding mode controller, generating an exponential convergence to a residual domain arbitrarily small. To smaller errors, it behaves as a parametric adaptation law. Other important advantages of the adaptation law in dual mode with respect to other adaptation laws or known robust control algorithms are listed below:

- Generation of continuous control signals,

- Improvement of the robustness of the system,

- Limitation of the values of the estimated parameters through a projection, which has the effect of reducing the effective gain of the controller when the tracking error increases (reducing the sensitivity to measurement noises). 


\section{A. High-Gain Observer Based Control Algorithm}

The desired articular trajectory denoted by $q_{d}(t)$ is supposed uniformly bounded, twice continuously differentiable with its two first derivatives $\dot{q}_{d}(t)$ and $\ddot{q}_{d}(t)$ also uniformly bounded. In order to obtain this trajectory, the Cerebellum Path Generator [12] based on adept cycle is used. It generates the desired cartesian trajectories, which will be the input for the direct geometric model of the robot [13]. This scheme is illustrated in the diagram of figure 2 :

\begin{tabular}{|c|c|c|c|c|}
\hline Cerebellum & $\begin{array}{l}\text { Desired } \\
\text { Cartesian } \\
\text { Trajectory }\end{array}$ & Par2 & $\begin{array}{l}\text { Desired } \\
\text { Articular } \\
\text { Trajectory }\end{array}$ & $\begin{array}{l}\mathbf{q}_{\mathrm{d}} \\
\dot{\mathbf{q}}_{\mathrm{d}}\end{array}$ \\
\hline $\begin{array}{c}\text { PATH } \\
\text { GENERATOR }\end{array}$ & & $\begin{array}{l}\text { GEOMETRIC } \\
\text { MODEL }\end{array}$ & & $\ddot{q}_{d}$ \\
\hline
\end{tabular}

Fig. 2. Generation of the desired articular trajectories

According to [16], the following errors are introduced:

$$
\tilde{q}=q_{d}-q, \dot{\tilde{q}}=\dot{q}_{d}-\dot{q}, s=\dot{\tilde{q}}+\lambda \tilde{q}, \dot{q}_{r}=\dot{q}_{d}-\lambda \tilde{q}
$$

where $\tilde{q}$ is the position error, $q_{d}$ is the desired joint position, $q$ is the measured joint position, $\dot{\tilde{q}}$ is the velocity error, $s$ is an auxiliary error, $\lambda$ is a positive constant, and $\dot{q}_{r}$ is the denominated 'reference velocity' [16],[17].

Assume that only the articular positions are measured (which is the case of the Par2 manipulator). Therefore the easiest way to compute the articular velocities consists in a numerical derivation. However, if the measured positions are noisy or do not have a good enough resolution, this technique will amplify the noise/quantization effect. In order to overcome this problem, an observer-based technique is proposed. It consists in estimating the joint velocities by means of a High-gain observer (HGO) [9], described by the following:

$$
\left\{\begin{array}{l}
\dot{\hat{x}}_{1}=\hat{x}_{2}+\frac{1}{\varepsilon} \eta_{1}\left(x_{1}-\hat{x}_{1}\right) \\
\hat{\hat{x}}_{2}=\frac{1}{\varepsilon^{2}} \eta_{2}\left(x_{1}-\hat{x}_{1}\right)-\ddot{q}_{d}+\hat{F}\left(\hat{x}, q_{d}, \dot{q}_{d}\right)+\hat{H}\left(\hat{x}_{1}, q_{d}\right) \tau^{s}
\end{array}\right.
$$

where

- $x_{1}$ represents the error of the system $\left(x_{1}=\tilde{q}\right)$ and $x_{2}$, its derivative,

- $\hat{x}_{1}$ and $\hat{x}_{2}$ represent the estimated states,

- $\varepsilon, \eta_{1}$ and $\eta_{2}$ are positive constants,

- $\hat{F}\left(\hat{x}, q_{d}, \dot{q}_{d}\right)=-D^{-1}\left(x_{1}, q_{d}\right)\left[C\left(x, q_{d}, \dot{q}_{d}\right)\left(x_{2}+\dot{q}_{d}\right)+\right.$ $\left.G\left(x_{1}, q_{d}\right)\right]$

- $\hat{H}\left(\hat{x}_{1}, q_{d}\right)=D^{-1}\left(x_{1}, q_{d}\right)$,

- $\tau^{s}$ is the saturated torque (to avoid the 'peaking phenomena').

The control architecture is illustrated in figure 3, and will be detailed in the following:

As illustrated in figure 3, the control law consists in the sum of three terms: an 'adaptive term', a 'smooth variable structure term' and a 'stabilizing term', and is given by:

$$
\tau=Y \hat{a}+\bar{d} \operatorname{Sat}(\alpha s)+K . s
$$

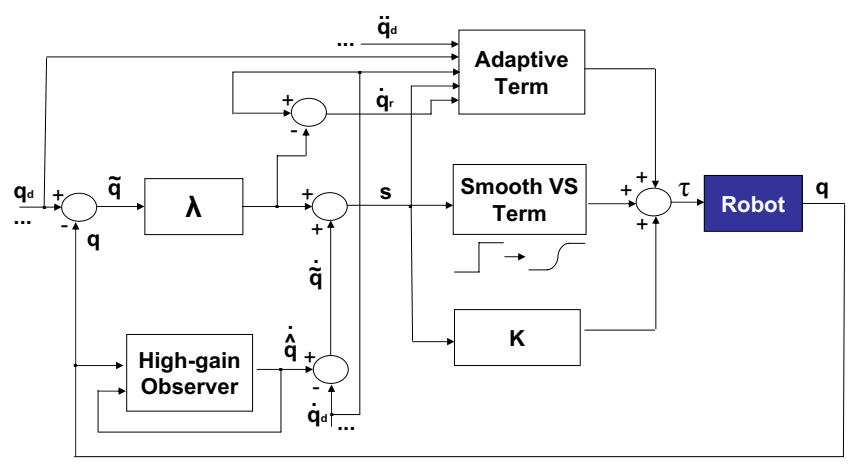

Fig. 3. Block diagram of the proposed control scheme

where

$$
\operatorname{Sat}(\alpha s)=\frac{\alpha s}{\|\alpha s\|+1}
$$

being $\bar{d}, \alpha$ and $K$ positive constants, Sat(.) is a continuous function with respect to its argument (with continuous partial derivatives and components limited to the interval $[-1,+1]$ ). The vector $\hat{a}$ represents an estimate of the unknown parameters of the system given by the vector $a$, and $Y$ is the regressor vector (based on the dynamic model of the system). The adaptation of the parameters is given by:

$$
\dot{\hat{\theta}}=-\sigma \hat{\theta}-\gamma_{s} Y
$$

where

$$
\hat{\theta}=\hat{a}-a_{\text {nom }}
$$

is the difference between the currently estimated values and the nominal values of the parameters. The variable $\sigma$ is given by the following:

$$
\begin{gathered}
\sigma=\left\{\begin{array}{cl}
0, & \text { if }\|\hat{\theta}\|<M_{\theta} \text { or } \sigma_{e q}<0 \\
\sigma_{e q}, & \text { if }\|\hat{\theta}\| \geq M_{\theta} \text { and } \sigma_{e q} \geq 0
\end{array}\right. \\
\sigma_{e q}=-\frac{\gamma_{S} Y \hat{\theta}}{\|\hat{\theta}\|^{2}}
\end{gathered}
$$

being $M_{\theta}$ the maximum possible value (supposed known) of the norm of the estimated deviation of the parameters with relation to their nominal values.

Let us now consider the dynamic model (2) with the estimates of the system parameters $\hat{a}$ :

$$
D(q, \hat{a}) \ddot{q}+C(q, \dot{q}, \hat{a}) \dot{q}+G(q, \hat{a})+f(q, \dot{q}, \hat{a})=\tau
$$

the regressor vector $Y=Y\left(q, \dot{q}, \dot{q}_{r}, \ddot{q}_{r}\right)$ can then be computed:

$$
D(q, \hat{a}) \ddot{q}_{r}+C(q, \dot{q}, \hat{a}) \dot{q}_{r}+G(q, \hat{a})+f(q, \dot{q}, \hat{a})=Y \hat{a}
$$

Note that in (12) we use $\dot{q}_{r}$ and $\ddot{q}_{r}$ instead of $\dot{q}$ and $\ddot{q}$. The key idea comes from eliminating undesirable steady-state position errors by restricting them to evolve on a sliding surface [16] such as in robust sliding mode control [2]. 
By neglecting the mass of the platform and the dry friction, these terms become $D(q, \hat{a})=I_{\text {motor }}+I_{\text {arm }}+I_{\text {forearm }}$, $f(q, \dot{q}, \hat{a})=f_{v}$ and:

$$
G(q, \hat{a})=-\frac{g}{2}\left(M_{1}+M_{2}\right)\left[\begin{array}{l}
L_{1} \cos \left(q_{1}\right) \\
L_{2} \cos \left(q_{2}\right)
\end{array}\right]
$$

where

$$
\begin{gathered}
I_{\text {motor }}=\left[\begin{array}{cc}
I_{m} & 0 \\
0 & I_{m}
\end{array}\right], \\
I_{\text {arm }}=\left[\begin{array}{cc}
I_{a} & 0 \\
0 & I_{a}
\end{array}\right], \\
I_{\text {forearm }}=\left[\begin{array}{cc}
L_{1}^{2 \frac{m_{2}}{2}} & 0 \\
0 & L_{2}^{2} \frac{m_{2}}{2}
\end{array}\right]
\end{gathered}
$$

By rewriting the dynamics of the system such that it has the form given in (12), one can find that:

$$
Y=\left[\begin{array}{cccc}
\ddot{q}_{1 r} & 0 & \dot{q}_{1 r} & -L_{1} \cos \left(q_{1}\right) \\
0 & \ddot{q}_{2 r} & \dot{q}_{2 r} & -L_{2} \cos \left(q_{2}\right)
\end{array}\right]
$$

and

$$
\hat{a}=\left[\begin{array}{c}
\hat{a}_{1} \\
\hat{a}_{2} \\
\hat{a}_{3} \\
\hat{a}_{4}
\end{array}\right]=\left[\begin{array}{c}
I_{m}+I_{a}+L_{1}^{2} \frac{m_{2}}{2} \\
I_{m}+I_{a}+L_{2}^{2} \frac{m_{2}}{2} \\
f_{v} \\
\frac{g}{2}\left(M_{1}+M_{2}\right)
\end{array}\right]
$$

\section{REAL-TIME EXPERIMENTAL RESULTS}

The objective of this section is to present real-time experimental results of the application of the proposed control scheme described in section III to the Par2 parallel manipulator described in section II. A PD controller was also implemented for a performance comparison with respect to the proposed controller.

The platform evolves in the $X O Z$ plane (being $X$ the horizontal axis and $Z$ the vertical axis). The desired cartesian trajectory to be tracked by the Par2 parallel robot is a usual 'pick-and-place' trajectory, and its parameters are described in table I. The parameters of the proposed control approach are summarized in table II.

TABLE I

PARAMETERS OF THE CARTESIAN REFERENCE TRAJECTORY

\begin{tabular}{|c|l|c|}
\hline Parameter & Description & Value \\
\hline$\left(x_{d_{i}}, z_{d_{i}}\right)$ & $\begin{array}{l}\text { Initial desired cartesian position } \\
\text { in the plane } X O Z\end{array}$ & $(-0.35 \mathrm{~m},-0.95 \mathrm{~m})$ \\
\hline$\left(x_{d_{f}}, z_{d_{f}}\right)$ & $\begin{array}{l}\text { Final desired cartesian position in } \\
\text { the plane } X O Z\end{array}$ & $(0.35 \mathrm{~m},-0.95 \mathrm{~m})$ \\
\hline$\dot{x}_{d}^{\max }$ & Maximum cartesian velocity & $8 \mathrm{~m} / \mathrm{s}$ \\
\hline$\dot{x}_{d}^{\max }$ & Maximum cartesian acceleration & $20 \mathrm{G}$ \\
\hline
\end{tabular}

This experimental scenario deals with the control of the parallel manipulator for one cycle of a pick-and-place trajectory, that is, the robot's platform has to go (as illustrated in figure 4) from the initial cartesian position $\left(x_{d_{i}}, z_{d_{i}}\right)$ to the desired final cartesian position $\left(x_{d_{f}}, z_{d_{f}}\right)$ and then return to
TABLE II

PARAMETERS OF THE CONTROL APPROACH

\begin{tabular}{|c||l|}
\hline \multicolumn{1}{|c|}{ Parameter } & Description \\
\hline$K_{p}=94.5$ & Proportional gain \\
\hline$K_{d}=2.1$ & Derivative gain \\
\hline$\lambda=25$ & Positive constant \\
\hline$K=2 I$ & Matrix gain \\
\hline $\bar{d}=2.5$ & Smooth variable structure gain \\
\hline$\alpha=0.05$ & Smooth variable structure slope \\
\hline$\varepsilon=0.002, \eta_{1}=\eta_{2}=1$ & HGO gains \\
\hline$M_{\theta}=0.25$ & $\begin{array}{l}\text { Maximum value of the norm of the adaptive } \\
\text { parameters' deviation }\end{array}$ \\
\hline$\gamma=0.3345$ & Adaptive gain \\
\hline$T_{s}=0.0005$ & Sampling time (s) \\
\hline$n=3$ & Number of cycles \\
\hline
\end{tabular}

the initial one $\left(x_{d_{i}}, z_{d_{i}}\right)$. The corresponding cartesian reference trajectory and the illustration of the robots movements are plotted in figure 4 .
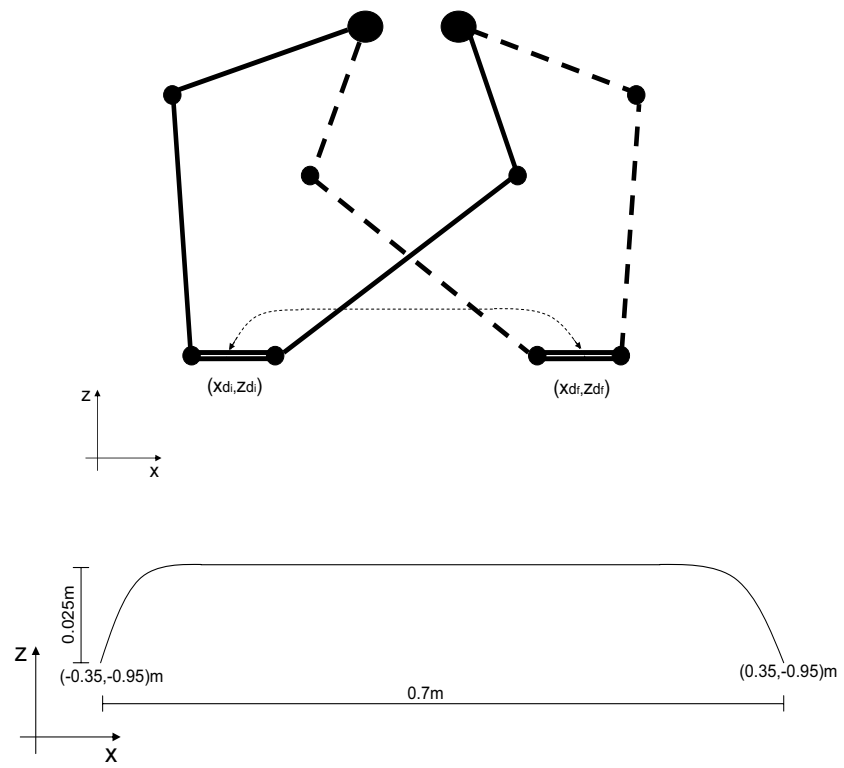

Fig. 4. Illustration of the robot's movements (top) and the desired cartesian trajectory $x-z$ in a larger scale (bottom)

The trajectory tracking obtained by the PD controller and by the DM controller for $20 \mathrm{G}$ is shown in figure 5 , the tracking errors are shown in figure 6, and the control inputs are shown in figure 7 . The real-time implementation of both controllers was made with a sampling time of $0.5 \mathrm{msec}$. In such scenario, the main control objective is to have the smallest tracking error possible, specially on the final positions of the trajectory. These positions occur at $t \in[0.125,0.175]$ (final desired position) and at $t \in[0.325,0.375]$ (back to the initial desired position).

By analyzing figures 5 and 6 , it is possible to notice that the DM controller has a considerably smaller tracking error than the PD controller during all the trajectories tracking, specially in the stop points, which is the most important objective of this experiment. The PD controller keeps the 

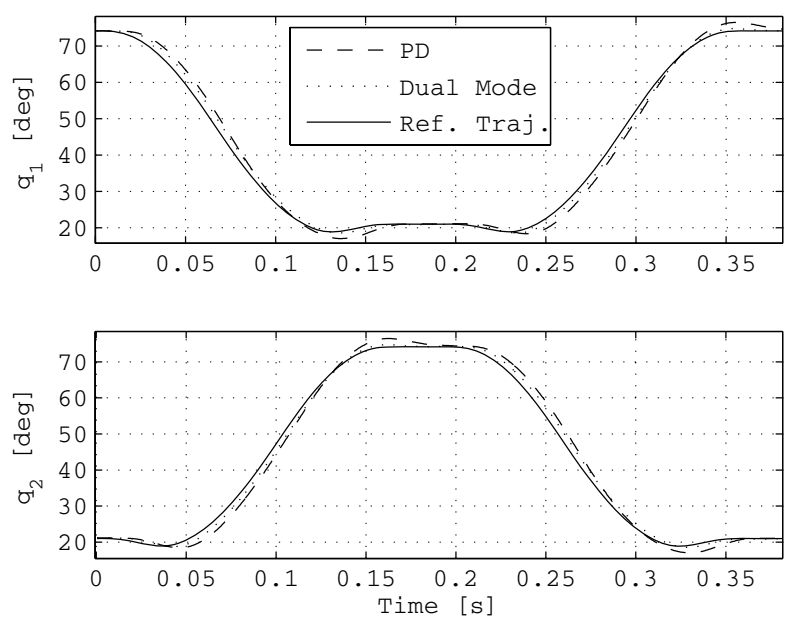

Fig. 5. Articular reference trajectory for 20G (solid), PD tracking (dashed), DM tracking (dots)
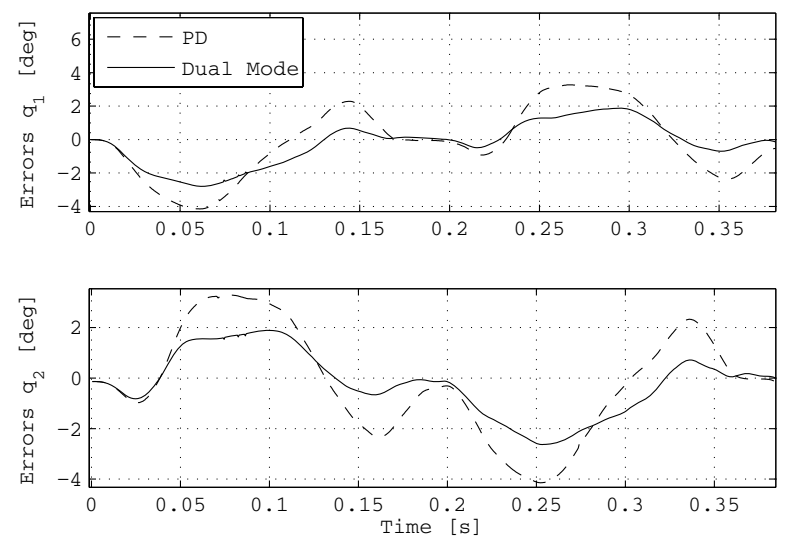

Fig. 6. Tracking errors for the PD controller (dashed) and for the DM controller (continuous)

tracking errors inside the limits of $\left[-4.15^{\circ}, 3.3^{\circ}\right]$ during all the trajectories, the DM controller keeps it inside the limits of $\left[-2.8^{\circ}, 1.9^{\circ}\right]$ (peak-to-peak improvement of approximately $37 \%$ ). And concerning the stop points, the PD controller generates errors inside $\left[-2.3^{\circ}, 2.3^{\circ}\right]$ while the DM controller generates errors inside $\left[-0.7^{\circ}, 0.7^{\circ}\right]$ (peak-to-peak improvement of approximately $70 \%$ ).

Concerning the control signals shown in figure 7 , it is possible to notice that the PD controller was delayed in comparison to the DM controller, and the amplitudes of both signals were roughly similar.

As it was noticed above, Par 2 is equipped with TPM50 Alpha motors of a maximum torque of 483 N.m, therefore according to figure 7 of the generated torques, we conclude that this bound is largely satisfied, but it should also be checked that the power requirement remains within the admissible limit.

To check the admissibility of the required power of the actuators, the idea is to plot their absolute torques versus
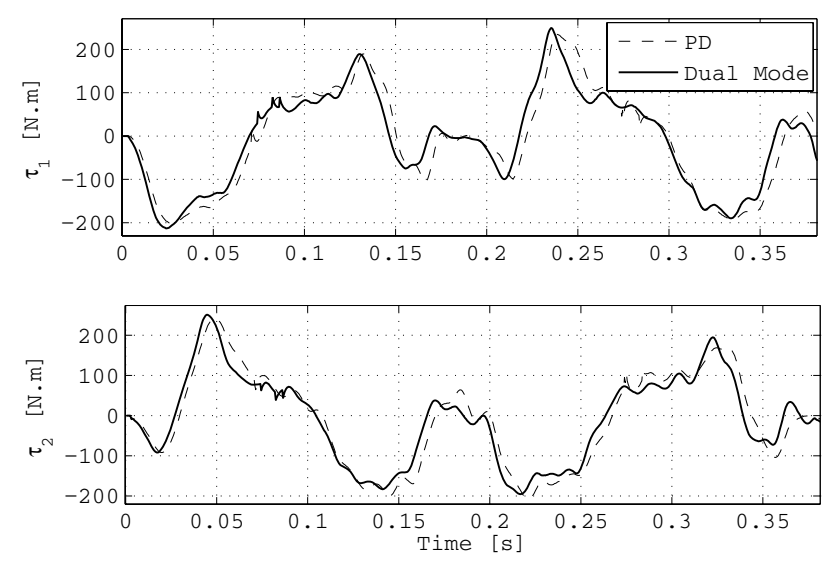

Fig. 7. Torques for the PD controller (dashed) and for the DM controller (continuous)
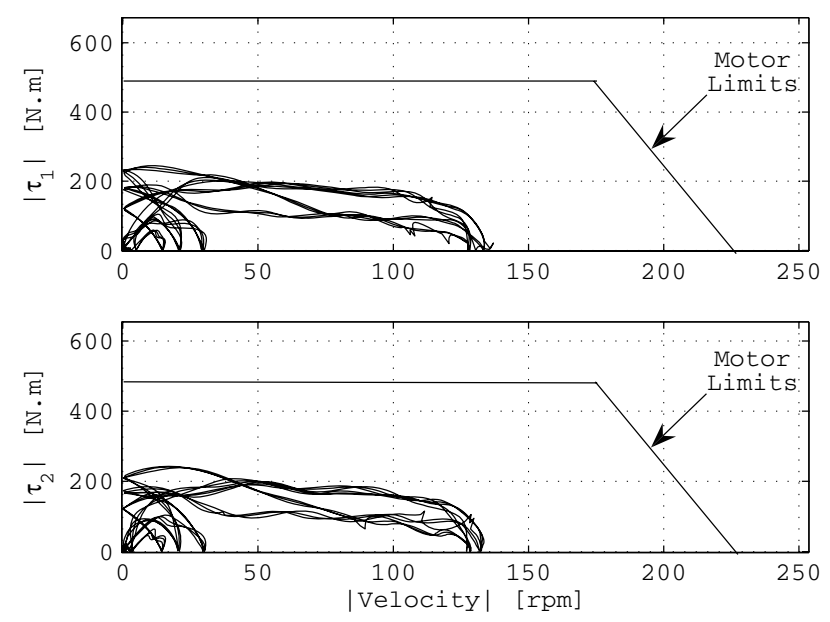

Fig. 8. Absolute torques of the actuators versus their absolute angular velocities for the PD controller, and admissible region

their absolute angular velocities, and to check if the obtained curves remain within the admissible region given by the manufacturer. These curves are plotted in figure 8 (for the PD controller) and in figure 9 (for the DM controller). According to the obtained results, it is easy to conclude that the required power of the actuators is admissible for both controllers.

The performance details of the two controllers are summarized on table III. The obtained movements of the robot while tracking the pick-and-place reference trajectory is illustrated by the video accompanying the paper.

\section{CONCLUSIONS AND FUTURE WORKS}

This paper deals with a nonlinear adaptive Dual Mode controller for the parallel manipulator (Par2), for a pick-andplace trajectory tracking with an acceleration of 20G. The obtained experimental results show that the Dual Mode (DM) controller has a considerably better performance than the conventional Proportional-Derivative (PD) controller. In the 

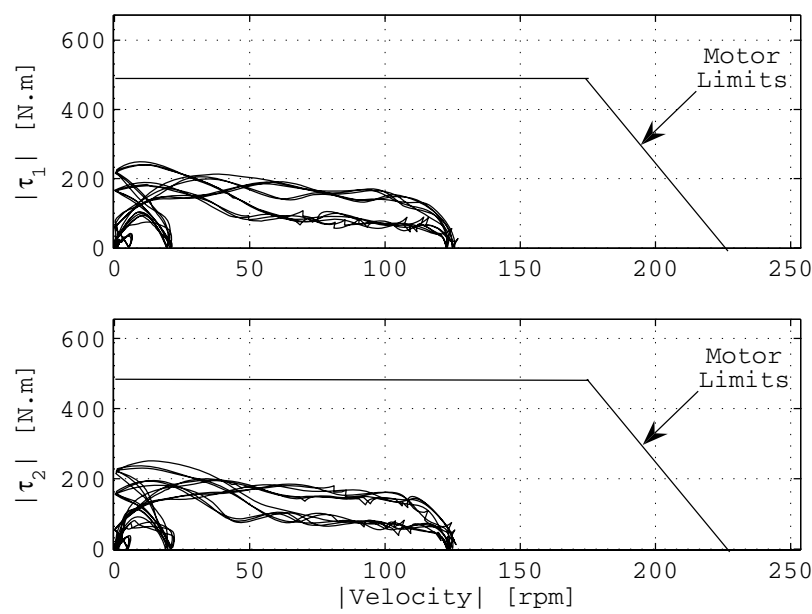

Fig. 9. Absolute torques of the actuators versus their absolute angular velocities for the DM controller, and admissible region

TABLE III

PERFORMANCE COMPARISON BETWEEN THE PROPOSED CONTROL APPROACH AND A CONVENTIONAL PD CONTROLLER

\begin{tabular}{|c||c||c|}
\hline Performance & PD & DM \\
\hline Error peaks & {$\left[-4.15^{\circ}, 3.3^{\circ}\right]$} & {$\left[-2.8^{\circ}, 1.9^{\circ}\right]$} \\
\hline Stop point errors & {$\left[-2.3^{\circ}, 2.3^{\circ}\right]$} & {$\left[-0.7^{\circ}, 0.7^{\circ}\right]$} \\
\hline \multirow{2}{*}{ Control signals } & PD controller delayed in comparison to the DM controller \\
\cline { 2 - 3 } & Roughly similar amplitude values \\
\hline
\end{tabular}

future, the proposed control approach will be evaluated for higher accelerations and also for different load conditions.

\section{REFERENCES}

[1] V. Bobal, J. Bohm, J. Fessl, and J. Machacek. Digital Self-Tuning Controllers. Springer Varlag, 2005.

[2] S. K. Spurgeon C. Edwards. Sliding mode control: theory and applications. CRC Press, 1998.

[3] R. Clavel. Delta, a fast robot with parallel geometry. International Symposium on Industrial Robots, pages 91-100, 1988.

[4] J. J. Craig. Adaptive Control of Mechanical Manipulators. AddisonWesley Publishing Company, 1988.

[5] A. A. Feldbaum. Optimal Control Systems. Academic Press, 1965.

[6] M. Honegger, R. Brega, and G. Schweitzer. Application of a nonlinear adaptive controller to a 6 dof parallel manipulator. Proc. IEEE Conf. Robotics Automat., 2:1930-1935, 2000.

[7] M. Honegger, A. Codourey, and E. Burdet. Adaptive control of the hexaglide, a 6 dof parallel manipulator. In In IEEE International Conference on Robotics and Automation, 1997.

[8] L. Hsu and R. R. Costa. B-mrac global exponential stability with a new model reference adaptive controller based on binary control theory. Contr. Theory Adv. Technol., 10(4):649-668, 2003.

[9] K. W. Lee and H. K. Khalil. Adaptive output feedback control of robot manipulators using high-gain observer. Int. J. of Contr., 67(6):869886, 1997.

[10] R. Monopoli. Adaptive control: The model reference approach. IEEE Trans. on Automat. Contr., 26(2):621-622, 1981.

[11] V. Nabat, M. de la O Rodriguez, O. Company, S. Krut, and F. Pierrot. Par4: very high speed parallel robot for pick-and-place. Proc. IEEE/RSJ Int. Conf. Intel. Robotics Systems., pages 553-558, 2005.

[12] Cerebellum Automation (part of Adept Technology Inc.). http://www.cerebellum-automation.com/addons.htm. 2009.

[13] F. Pierrot, C. Baradat, V. Nabat, O. Company, S. Krut, and M. Gouttefarde. Above $40 \mathrm{~g}$ acceleration for pick-and-place with a new 2-dof pkm. In Proc. IEEE Conf. Robotics Automat., Kobe, Japan, 2009.
[14] L. Sciavicco and B. Siciliano. Modeling and control of robot manipulators. McGraw Hill, New York, 1996.

[15] J. S. Shamma and M. Athans. Analysis of gain scheduled control for non linear plants. IEEE Trans. on Automat. Contr., 35(8):898-907, 1990.

[16] J.J. Slotine and W. Li. Adaptive manipulator control: A case study. IEEE Trans. on Automat. Contr., 33(11):995-1003, 1988.

[17] J.J. Slotine and W. Li. Applied Nonlinear Control. Prentice Hall, 1991.

[18] M. Spong and M. Vidyasagar. Robot Dynamics and Control. John Wiley \& Sons, New York, 1989. 